\title{
Neurological gait disorders in childhood
}

\section{$\underline{\text { Authors }}$}

\section{Martin Smith and Manju A Kurian}

Martin Smith (corresponding author)

Consultant Paediatric Neurologist

John Radcliffe Hospital

Oxford

$\mathrm{OX}_{3}$ 9DU

E mail: martinsmith3@nhs.net

Manju A Kurian

NIHR Research Professor; Consultant Paediatric Neurologist

Developmental Neurosciences

UCL Great Ormond Street Institute of Child Health

30 Guilford Street

London

$\mathrm{WC}_{1} \mathrm{~N}_{1} \mathrm{EH}$

E mail: manju.kurian@ucl.ac.uk

\section{$\underline{\text { Abstract }}$}

There are an enormous number of neurological illnesses that can manifest with gait disturbance in childhood. Whilst experience and clinical acumen are helpful in diagnosing these disorders some basic principles in assessment and diagnosis are helpful in determining the phenomenology, time course, and neuro-anatomical localisation. In this review we focus on some of the more common movement disorders resulting in inserted postures (including spasticity and dystonia), inserted movements (including chorea and myoclonus), and impairment of motor control (including ataxia and neuro-muscular disorders). A number of case studies are included to illustrate the factual descriptions.

\section{$\underline{\text { Key words }}$}

Gait disorders in childhood Spasticity

Dystonia

Chorea

Ataxia

Myoclonus 


\section{Introduction (A)}

Although many other neurological impairments can be a far greater barrier to independence, gait disorders remain an important group of presentations in child neurology. It is useful to be able to recognise the key features in presentation, to guide strategies for diagnosis and therapy.

The neurological building blocks of successful ambulation include strength, balance and planning of complex movement. Sensory input including vision, vestibular and proprioceptive feedback are also integral. It follows that ambulation can be threatened by weakness, poor balance, poor motor planning and control, and sensory impairments. These difficulties can be continually present, or episodic.

In this article, we focus primarily on some of the more common movement disorders resulting in inserted postures (including spasticity and dystonia), inserted movements (including chorea and myoclonus), and impairment of motor control (including ataxia and neuro-muscular disorders), which can overlap in many individuals. A number of case studies are included to illustrate the factual descriptions.

\section{Spasticity (B)}

Spasticity is defined as a velocity-dependent increase in muscle tone. It typically results in co-contraction of antagonist muscle pairs, with a bias towards greater involvement of the upper limb flexors and lower limb extensor muscle groups. Spasticity of the lower limbs tends to lead to hip extensor tightness, reduced knee flexion during swing through, and equinus posture due to excessive contraction of gastrocnemius. This results in forefoot contact, and the so-called waddling gait as circumduction of the hip compensates to allow swing through. Spasticity may occur following lesions to the brain or spinal cord (pyramidal tract lesions), but can also occur in non-lesional disorders such as hereditary spastic paraparesis.

Investigations should always include imaging of the brain and spinal cord, and if normal may require further investigations e.g. metabolic investigations, and genetic screen for hereditary spastic paraparesis.

There are many therapeutic options to reduce spasticity in specific or wider muscle groups. These include drugs (e.g. oral Baclofen), localised neuromuscular blockade (Botulinum neuro-toxin), and neurosurgical procedures (e.g. selective dorsal rhizotomy and intra-thecal Baclofen delivery). Physiotherapy is at least as important as all these options, and the absence of physiotherapy renders the medical interventions nearly futile.

Whenever these treatments are employed it is important to have clear functional goals, and always remember that spasticity is one part of the upper motor neuron syndrome, along with weakness and poor motor planning. Spasticity reduction in itself 
may not always lead to improved function, and may even worsen function, particularly in the context of underlying weakness/instability.

\section{Case study $(C)$}

A 14-year-old female is referred with a gait disorder. She was born prematurely, at 28 weeks gestation, but attained her motor milestones within the normal range. She has high functioning autistic spectrum disorder and anxiety. There is no relevant family history.

Examination reveals unequivocal upper motor neuron signs, predominantly in the lower limbs. MRI scan reveals white matter abnormalities adjacent to the lateral ventricles, consistent with white matter injury of prematurity.

Genetic testing revealed a mutation in the $K_{I} F_{5} A$ gene, associated with Hereditary Spastic Paraparesis type 10. The history of prematurity and PVL was incidental to her gait disorder.

\section{Dystonia (B)}

Dystonia has similarities to spasticity, in leading to co-contraction of antagonistic muscle groups. However, the velocity-dependent element is absent, the disorder is more fluctuant with variability in tone, can be task specific, and more likely to lead to twisting postures.

Gait disorders in dystonia may appear at times frankly bizarre, due to the predominance of extensor activity and twisting postures. As a general rule, the ankles will demonstrate equinovarus posture, but there is a greater range of abnormalities than seen in spasticity. Moreover, the task specific element can be a source of confusion in distinguishing between dystonia and functional gait disorders. For example, it is entirely possible for an individual with dystonia to be unable to walk forward without assistance, but able to walk backwards unaided, or even ride a bicycle.

Dystonia is typically associated with lesions of the thalami and basal ganglia (extrapyramidal tracts) but can also occur in non-lesional, usually genetic, disorders e.g.DYT1 and DYT5 (dopa-responsive dystonia).

The investigation of dystonia should be targeted to the clinical context. An MRI brain scan is invariably required, and further investigations may include:

- Copper and caeruloplasmin (Wilson's disease)

- Blood film (for acanthocytes)

- TSH, T3 and T4 (Allan-Hernon Dudley syndrome)

- Urate (Lesch-Nyhan syndrome) 
- Paired blood and CSF glucose (GLUT1 deficiency)

- CSF neurotransmitters (disorders of dopamine/serotonin synthesis, metabolism or transport) and lactate (mitochondrial disorders)

- Other metabolic tests - plasma lactate, manganese, biotinidase, very long chain fatty acids, lysosomal screen, vacuolated lymphocytes, acylcarnitine profile, transferrin isoelectric focusing

- Urine organic acids, creatine to creatinine ratio

- CGH microarray (for copy number variants, deletions and duplications encompassing disease-causing genes) and multigene panels for monogenic dystonia (e.g. DYT1, DYT11, PANK2/PLA2G6 and other disorders of neurodegeneration with brain iron accumulation, $K M T_{2} B$ and other genes causing complex dystonia phenotypes)

- Neurophysiology should not be required to diagnose pure dystonia, but some disorders demonstrate a mixed pattern of dystonia and lower motor neuron signs

Therapeutic options to manage dystonia include medication (e.g. Trihexyphenidyl, Gabapentin, Clonidine), Botulinum toxin, ITB and deep brain stimulation. The latter is particularly effective in primary dystonia with normal imaging, but also offers some degree of benefit in secondary dystonia, notably Pantothenate Kinase Associated Neurodegeneration. The ketogenic diet may be considered in individuals with GLUT1 deficiency. A trial of Levodopa can significantly ameliorate or even abolish motor symptoms in patients with Segawa's disease (DYT5-dystonia) and other doparesponsive disorders, and should be considered first line in patients with dystonia of undetermined aetiology.

Some forms of dystonia may be intermittent, and can be characterised as either movement related (kinesogenic), not-related to movement (non-kinesogenic), or specifically related to sustained exercise (Lance type). Paroxysmal kinesogenic dystonia is triggered by sudden movement, and episodes are short lasting on average, approx. $1 \mathrm{~min}$. They are associated with mutations in the PRRT2 gene, and typically respond very well to low dose Carbamazepine. Non-kinesogenic dystonic episodes tend to last much longer (up to several hours), and may be triggered by caffeine or alcohol. They respond less well to Carbamazepine, but may respond to benzodiazepines. Exercise induced dystonia show mixed responses to these medications.

\section{Case study (C)}

A 6-year-old girl is referred for advice on the management of cerebral palsy. On closer questioning she was born at term following an uneventful pregnancy, in good condition, and did not display any feature of neonatal encephalopathy. She had never walked unaided, but had been able to walk with the help of one from 3 years of age. 
MRI brain and spine were normal. Examination findings were suggestive of bilateral lower limb spasticity, although plantar responses were flexor (downgoing).

The specific question parents asked is "why doesn't she have cerebral palsy in the morning, doctor?".

The history of fluctuation, together with the unremarkable neonatal history and normal imaging, suggested the diagnosis of dopa-responsive dystonia (Segawa disease or DYT5). She was given an empirical trial of low dose L-Dopa $(2 \mathrm{mg} / \mathrm{kg} / \mathrm{day})$, and was walking normally within one week. Subsequent genetic testing revealed a mutation in the $\mathrm{GCH}_{1}$ gene. Neurotransmitters were not measured, but would be expected to show low neopterin, low $\mathrm{BH}_{4}$, low HVA, low/normal 5-HIAA, although for some patients, the CSF profile can be (near) normal.

She remained with near normal mobility until transition to adult services. The only residual abnormality was very mild ankle varus. However, she was susceptible to anxiety and depression (which is reported in this condition, and possibly related to cerebral serotonin deficiency) which was not corrected by L-Dopa replacement.

\section{Case study (C)}

A 9-year-old girl is referred with in-turning of the left ankle. Over the following 12 months this progresses, with involvement of all four limbs. She becomes nonambulant. Her facial muscles are spared. MRI scan and baseline metabolic investigations are normal. Genetic testing reveals the heterozygous common mutation (GAG deletion) in the TOR $1 A$ (DYT1) gene.

She proceeds to implantation of a deep brain stimulator, with bilateral electrodes positioned in the globus pallidus interna. Initial improvement is modest, but over 6 months she regains entirely normal function, which is maintained at last follow up over a decade later.

\section{Case study (C)}

A 15-year-old male is referred with walking difficulties. For 6 months previously he had had some personality change attributed to teenage moodiness. Examination revealed generalised dystonia. MRI scan revealed high signal with the basal ganglia. There was no obvious Kayser-Fleisher ring to routine inspection. However, he was found to have low values of serum copper and caeruloplasmin, and a mutation in the $A T P_{7} B$ gene, confirming the diagnosis of Wilson's disease.

He was treated with chelation therapy with Trientene and Zinc, with satisfactory gradual reduction in urinary copper values. However, his dystonia continued to progress and he became non-ambulant. 
Comment: Early diagnosis is crucial in Wilson's disease, although chelation therapy is not always effective in preventing further deterioration of physical signs.

\section{Case study (C)}

A 6-year-old male is referred with a gait disorder superimposed on mild global developmental delay. Examination findings are non-specific, but appear to be a mixed picture of extra-pyramidal signs and weakness. MRI brain revealed cerebellar atrophy.

Whole exome sequencing identifies bi-allelic mutations in the PLA2G6 gene. Over the following 3 years he becomes non-ambulant, with cortical visual impairment and worsening cognitive ability. DBS is considered but declined, and he is listed for an ITB pump to manage worsening dystonia.

\section{Case study (C)}

A 14-year-old male is referred with an intermittent gait disorder. He was playing as goalkeeper for a football team, but has recently stopped as he would become frozen or locked when he needed to rush out towards on onrushing attacker.

Routine neurological examination was unremarkable, but when asked to stand and run suddenly he developed generalised dystonia lasting approx. 1 minute.

The diagnosis of paroxysmal kinesogenic dystonia led to the prescription of low dose Carbamazepine $(5 \mathrm{mg} / \mathrm{kg} /$ day), on which he became entirely asymptomatic.

Subsequent genetic testing identified a mutation in the PRRT2 gene.

\section{Chorea and athetosis (B)}

Chorea produces involuntary, apparently random, high frequency inserted movements. Athetosis is similar, but of lower intensity, almost writhing, and can be likened in some respects to a cat's tail in the relatively languid appearance of the movements.

The gait manifestations seen in chorea are primarily due to the breakdown of coordinated movement, although can be exacerbated by co-existing dystonia and weakness.

Relatively common causes include neonatal hypoxic-ischaemic injury (sometimes labelled as dyskinetic cerebral palsy), hyper-bilirubinaemia, para-infectious or poststreptococcal syndromes, and rare but increasingly recognised disorders such as NMDA receptor antibody encephalitis. Benign hereditary chorea, is associated with mutations in the $\mathrm{NKX}_{2}-1$ gene which encodes the Thyroid Transcription Factor 1 (TITF1). 
$A D C Y-5$ related dyskinesias typically present with axial hypotonia and delayed motor and/or language milestones during infancy, associated with early onset chorea with a generalised distribution, classically also involving the facial muscles and peri-oral region.

Mutations in GNAO1 were initially reported in association with early infantile epileptic encephalopathy. Subsequently the recognised phenotype has widened to include a hyperkinetic movement disorder, with exacerbations triggered by fever and other intercurrent illness.

Treatment may be targeted against the underlying disorder (e.g. plasma exchange for NMDAR Ab encephalitis), but medication to suppress chorea may include Levetiracetam, Tetrabenazine, Sodium Valproate and Sulpiride.

Deep brain stimulation has proven to be partially effective in some conditions. For example, bilateral GPi DBS can substantially improve the frequency and severity of life-threatening exacerbations in individuals with GNAO1 mutations, although the baseline movement disorder is largely unchanged.

\section{Case study $(C)$}

A 14-year-old female presented to hospital with headaches and a first seizure. GCS was 15, with no focal neurology. A routine CT scan was normal. CSF examination revealed 55 white cells, no red cells, and PCR was positive for herpes simplex. She was treated with 3 weeks of iv acyclovir. After 2 weeks she deteriorated substantially, developing a choreiform movement disorder. She became encephalopathic, and required tube feeding. She was incontinent.

Immunological testing revealed antibodies against NMDA receptors. Ovarian teratoma and other neoplastic lesions were not found. She was treated with high dose steroids, IVIG, and plasma exchange. She improved only very slowly, but by 9 months she was able to be discharged home. She returned to school 12 months after the onset of her illness, and subsequently achieved to GCSE qualifications.

\section{Myoclonus (B)}

Myoclonus is the fastest of the movement disorders, typically producing "electric shock" type movements or jerks (positive myoclonus) of 10-50ms duration, although spinal or brain stem myoclonus may last up to 200ms. Negative myoclonus refers to sudden, brief relaxation of muscles, and may result in drop attacks.

It can be caused by lesions within widely dispersed parts of the central nervous system, including the cortex, sub-cortex, brain stem and spinal cord. Important features to extract in the history and examination include whether it occurs at rest, during action, or is stimulus driven, and whether it is irregular or rhythmic. 
As with chorea, the gait manifestations seen in myoclonus are primarily due to the interruption of smooth co-ordinated movement, although can be exacerbated by coexisting dystonia etc.

Myoclonus can be benign, but is sometimes a feature of progressive neurodegenerative disorders. A key distinction is between epileptic and non-epileptic myoclonus, which is relatively simple by means of ictal EEG. Epilepsy syndromes with myoclonus include juvenile myoclonic epilepsy, myoclonic absence and myoclonic astatic epilepsy. Progressive myoclonic epilepsy is a feature of neuronal ceroid lipofuscinosis, mitochondrial disease, Unverricht-Lundborg disease, sialidosis and Lafora body disease.

Non-epileptic myoclonus is seen in dystonia-myoclonus syndrome (DYT11), and opsoclonus-myoclonus syndrome. Non-epileptic myoclonus is often pharmacoresistant, but options can include piracetam, levetiracetam, and topiramate.

\section{Case study $(C)$}

A 5-year-old girl is seen in clinic with a several years history of hand tremor and a mild gait disorder. Her paternal grandmother had suffered a persistent tremor since childhood, attributed to meningitis in infancy. The child's father was healthy.

Examination revealed marked pectoral myoclonus, with mild dystonic posturing of the outstretched hands. Her gait was only mildly abnormal, with only a hint of dystonia.

Genetic testing confirmed a pathogenic heterozygous mutation in the epsilonsarcoglycan gene (DYT11) in all three family members.

There was no benefit in the upper limb tremor from oral medication (including levetiracetam, piracetam, and trihexyphenidyl). Deep brain stimulation was considered but declined by the family.

Comment: DYT11 commonly displays the genetic phenomenon of maternal imprinting, with inactivation of the maternal allele. When inherited from the mother, the disease manifests in only $5-10 \%$ of individuals. When inherited from the father, approx. $90 \%$ will be affected.

\section{$\underline{\operatorname{Ataxia}(\mathrm{B})}$}

Ataxia is defined as an inability to generate a normal or expected voluntary movement trajectory that cannot be attributed to weakness or involuntary muscle activity about the affected joints. Typically this manifests as a broad based gait disorder due to impaired balance and co-ordination, and may be due to pathology affecting cerebellar motor control or impaired proprioception. MRI brain imaging is mandatory in all new onset cases. 
Ataxia can be classified by acute, or chronic onset, and whether or not it is stable, progressive or episodic. Examples of acute non-progressive ataxia include acute cerebellitis (which is typically para or post infectious, and may follow Herpes Zoster), and Guillain-Barre syndrome (including the Miller Fisher variant). Chronic progressive disorders include ataxia telangiectasia, and Friedreich's ataxia.

Ataxia telangiectasia (AT) is important to recognise early, to avoid un-necessary exposure to ionising radiation. However, the characteristic ocular telangiectasia are rarely evident in pre-school years. It is therefore important to measure serum alphafeto protein levels in a child with unexplained ataxia or dystonia.

Ataxia with oculomotor apraxia type 1 has a similar neurological phenotype to AT, although the AFP is not elevated. Most cases are related to mutations in the APTX gene. AOA types 2 and 3 are also described.

The cardinal features of Friedreich's ataxia are the combination of gait ataxia, axonal neuropathy, areflexia, extensor plantar responses, cardiomyopathy and diabetes. There is no currently effective treatment, although Idebenone is often used empirically.

Other rare causes of ataxia which are potentially amenable to treatment, include ataxia with vitamin E deficiency, abetalipoproteinaemia, cerebrotendinous xanthomatosis, Refsum's disease, and Hashimoto encephalopathy.

There are two well described forms of episodic ataxia, although advances in genetic technologies have resulted in an increasing number of reported genetic episodic ataxias. In type 1 , the ataxia is usually brief, lasting seconds to minutes. Myokymia may be present between ataxic episodes. Triggers can include anxiety, excitement and fever. Most cases are related to heterozygous mutations in KCNA1. Acetazolamide may be helpful, but benefit is not always sustained.

In EA type 2 episodes may last hours to days, although some children will present with chronic progressive ataxia. Most cases are related to mutations in the $C A C N A \mathrm{~A}$ gene. In type 2, the episodes can last hours to days, and may also respond to Acetazolamide.

Mutations in $A T P_{1} A_{3}$ are associated with cerebellar ataxia, areflexia, pes cavus, optic atrophy, and sensori-neural hearing loss (CAPOS syndrome). ATP1A3 variants are also reported in both rapid-onset dystonia parkinsonism, and alternating hemiplegia of childhood.

\section{Case study $(C)$}

A 2-year-old boy is referred with motor delay and speech delay. He has recently started to walk but with a persisting broad-based gait. Both parents appear fit and well, but on closer questioning father admits to episodic difficulties with balance. On occasions, he has been refused entry to pubs as he appeared drunk, even before he had 
consumed any alcohol. He has always attributed this to tiredness, and had never sought medical advice.

Genetic investigations identified that both father and son have mutations in the $C A C N A 1 A$ gene. Both were offered a trial of acetazolamide. Father reported complete resolution of his symptoms, but unfortunately the child did not show any improvement on the maximum dose tolerated.

\section{Neuromuscular weakness (B)}

It is always important to bear in mind that disorders of the peripheral nervous system may impair gait, whether by weakness and/or impaired sensation.

Anatomically they can be considered disorders affecting the anterior horn cell (e.g. spinal muscular atrophy), peripheral nerve (e.g. Charcot-Marie-Tooth syndrome), neuromuscular junction (e.g. myasthenia gravis), or muscle (e.g. Duchenne muscular dystrophy). Typically these disorders will demonstrate weakness, wasting, and reduced or absent reflexes. Romberg's sign may be positive if the dorsal columns are involved.

The classical picture of a high stepping gait, due to weakness of ankle dorsiflexion, is commonly seen in peripheral neuropathies. This can often be heard as well as seen, with a slapping sound as the foot makes contact with the ground.

Investigations will usually include creatinine phosphokinase, nerve conduction studies and EMG, and more detailed genetic investigations targeted at the specific concerns.

\section{Functional gait disorders (B)}

This important group of disorders, sometimes also known as medically unexplained or psychogenic illness, commonly present to both Paediatric and Adult Neurology services. Most experienced clinicians will make an early diagnosis based on features that include fluctuation and inconsistencies, pattern and distribution of the movement disorder, in the absence of any plausible neuro-anatomical basis. However, a reasonable number of baseline investigations are usually required to reassure the child and family.

Whilst it is sensible to remain open-minded, it is important to be honest and open with the young person and family when the disorder appears unlikely to have an organic explanation. Detailed investigations for implausible diagnoses may delay or erode the prospects of recovery.

Many young people will respond to a holistic rehabilitation regime, which should ideally include a clinical psychologist. However, a significant minority can remain entrenched in a medical model of long term neurodisability. 


\section{$\underline{\text { Summary (A) }}$}

There are an enormous number of neurological illnesses that can manifest with gait disturbance. In this review we have attempted to describe some of the more common disorders. There is no substitute for experience in diagnosing these disorders, but basic principles in assessment and diagnosis include a careful history and examination to understand the phenomenology, time course, and neuro-anatomical localisation. Investigations will almost always include imaging of the brain and spinal cord, with further investigations targeted appropriately towards the differential diagnosis.

A proportion of disorders will be either self-limiting or amenable to a range of medical interventions, but many will prove to be chronic or progressive disorders, which require a holistic approach to caring for the young person and family.

\section{Further reading (B)}

Carecchio and Mencacci. Emerging monogenic complex hyperkinetic disorders. Curr Neurol Neurosci Rep 2017: 17:97

De Souza PVS, et al. Hereditary spastic paraplegia: clinical and genetic hallmarks. Cerebellum 2017: 16(2): 525-551

Kurian MA, Dale RC. Movement Disorders Presenting in Childhood. Continuum (Minneap Minn). 2016; 22 (Movement Disorders): 1159-85

Ramirez-Zamora A, et al. Treatable causes of cerebellar ataxia. Movement disorders 2015; 30: 614-623

Silveira-Moriyama L et al. Phenotypes, genotypes, and the management of paroxysmal movement disorder. Dev Med Child Neurology 2018; 6o(6): 559-565 